\title{
Glioblastoma and Glioblastoma with Oligodendroglial Component: a Histological and Prognostic Overview Runing Head: pathology and GBM and GBMO
}

\author{
Ravindra Pramod Deshpande ${ }^{1}$, Chandrasekhar YBVK ${ }^{2}$, Manas Panigrahi² ${ }^{2}$ S Satish Rao ${ }^{3}$ and Phanithi Prakash \\ Babu $^{1 *}$ \\ ${ }^{1}$ Department of Biotechnology and Bioinformatics, School of Life Sciences, University of Hyderabad, India \\ ${ }^{2}$ Department of Neurosurgery, Krishna Institute of Medical Sciences, Secunderabad, India \\ ${ }^{3}$ Department of pathology, Krishna Institute of Medical Sciences, Secunderabad, India
}

Received: 制: September 25, 2018; Published: 制: October 04, 2018

*Corresponding author: P Prakash Babu, Department of Biotechnology and Bioinformatics, School of Life Sciences, University of Hyderabad, Hyderabad-500046. Telangana state, India

\begin{abstract}
Glioblastoma (GBM- GIV astrocytoma) represents the most noted neoplasms of the brain usually reported with dismal prognosis. The term glioblastoma with oligodendroglial component (GBMO) represents the present of oligodendroglial foci in glioblastoma tumors. GBMOs are usually represented with better therapeutic response and concomitant prognostic outcome. We have observed nearly 610 cases of various pathologic grades of astrocytoma from 2009 to 2014 and found the oligodendroglial component among the 40 cases. Nearly in all the cases of GBM and GBMOs received complete resection and was followed by standard therapeutic regime of radiotherapy and chemotherapy with temozolomide. The survival pattern was observed in patients diagnosed with GBM and GBMO. We have looked for the presence of MIB1, p53 and necrosis pattern in selected GBMO sub group. We observe nearly 54\% of GBMOs stained positive with p53. Necrosis and MIB1 was observed in most of the GBMO cases. These was statistically significant difference among survival of glioblastoma patients' and GBMOs. Glioblastoma multiformae patients with oligodendroglial component was observed to have median survival of 16 months while with GBM it was observed to be 12 months. GBMO were found to have significantly longer survival than glioblastoma patients' and respond well to chemo and radiotherapy.
\end{abstract}

Keywords: Glioblastoma; GBMO; P53; Mib1; Survival

Abbreviations: GBMO: Glioblastoma with Oligodendroglial Component; WHO: World Health Organization; ICE: Institutional Ethics Committee; KFRC: KIMS Foundation Research Centre

\section{Introduction}

Glioblastoma are recognized as most aggressive and widespread malignancies of the brain. Irrespective of the multimodal treatment regime, prognosis still remains dismal. However, the clinical course appears to be heterogeneous ranging from 3 to 18 months [1,2]. The discrepancy in the prognosis is mainly attributed to few factors as age and presence of oligodendroglial component [2]. Necrosis and older age is often correlated with shorter survival in clinical cases [1]. Glioblastoma with oligodendroglial component (GBMO) are high grade astrocytic neoplasms with discrete oligodendroglial differentiation areas displaying necrosis [1]. Few studies have claimed that glioblastoma with oligodendroglial component display better prognostic pattern, yet this hypothesis remains controversial [3-8]. GBMOs have been reported to have distinct genetic alterations and clinical behavior and displays distinct IDH1 mutations along with $1 p / 19 q$ co deletion $[9,10]$. In present study, we have observed the molecular signatures of p53 and MIB1 in GBMO patients. Further, we have also seen for the presence of necrosis pattern and prognostically evaluated the GBMO cases and compared it with survival of GBM patients.

\section{Materials and methods}

\section{Selection of Patients}

The data of diagnosed clinical cases was collected from Krishna Institute of Medical Sciences, Secunderabad, India from 20092014. Upon initial MRI diagnosis, the patients were followed for surgical resection. The resected tissues were followed for the histopathological diagnosis based on the criterion World Health Organization (WHO) as cellular proliferation, mitotic index, polymorphic nuclei and the presence of necrosis. 610 cases were found to be diagnosed with astrocytoma, out of which 40 were found with glioblastoma with oligodendroglial component. The selected patients were not having previous history of low grade 
tumors and were followed up closely to obtain the survival data. All the patients were under went at near total or subtotal resection and confirmed by MRI imaging.

\section{Histological Diagnosis}

Out of total 610 cases, the glioblastoma with oligodendroglial component was confined to 40 cases (6.5\%). The paraffin embedded tissue sections of the resected tissues were observed for the presence of oligodendroglial tumor cells with characteristic pattern of round nuclei, honeycomb appearance and cytoplasmic swelling. The morphological appearance was confirmed by the two independent pathologists.

\section{Statistical Analysis}

The overall survival pattern of patients diagnosed with glioblastoma and oligodendroglial component were studied by Kaplan-Meier statistics. The comparison between the survival of GBM and GBMO patients was performed with log-rank test, $\mathrm{p}<0.05$ was considered as statistically significant.

\section{Results}

The details of the GBMO patients are given in Table 1. There were 40 cases we studied in a sub-group. Glioblastoma with oligodendroglial component constituted $21 \%$ of total glioblastoma cases and $6.5 \%$ of total astrocytoma cases. Age of patient's was found to be in range of $16-63$ years (male $=26$, females $=14$ ). The tumor location was found to be dominated by frontal and temporal lobes. Of total GBMO cases, tumor was found to be located in 13 cases at frontal lobe, in 16 cases at temporal lobe, in 6 cases at parietal lobe and in 5 cases at occipital lobe. Similar therapeutic regime of surgical resection followed by radiation therapy and chemotherapy with temozolomide was followed. The GBMO patients were closely followed up for the survival and tumor recurrence and was confirmed by the MRI imaging. Further, the p53, MIB1 and necrosis pattern was also observed (Figure 1). Out of 40 cases, 38 were found to be positive with MIB1 staining. In one case, the details were not available and one case was found negative. In 22 cases, the p53 protein was stained positive while in 8 cases negative staining was observed. The details were not available with 10 cases. Necrosis was found to be prevalent in 34 cases while not evident in 4 cases. The details of necrosis were not available in total of 2 cases. The follow up details clearly reveal that the GBMO patients are favored in survival over the GBM patients (Figure 2). The median survival of 16 months was observed in GBMO patients while 12 for the GBM patients (Figure 3). There was statistically significant difference observed among the survival of GBMO/GBM patients.

Table 1: Clinico pathological and prognosis details of GBMO patient's.

\begin{tabular}{|c|c|c|c|c|c|c|}
\hline Sr no & Age/sex & Tumor location & MIB 1 & Necrosis & P53 & Survival (in months) \\
\hline 1 & & Frontal Lobe & + & + & + & 15 \\
\hline 2 & & Frontal Lobe & + & + & + & 11 \\
\hline 3 & & Frontal Lobe & + & + & - & 13 \\
\hline 4 & & Frontal Lobe & + & + & + & 10 \\
\hline 5 & & Frontal Lobe & + & + & - & 28 \\
\hline 6 & & Frontal Lobe & + & + & + & 26 \\
\hline 7 & & Frontal Lobe & + & + & - & 31 \\
\hline 8 & & Frontal Lobe & + & + & + & 26 \\
\hline 9 & & Frontal Lobe & + & + & n.a. & 26 \\
\hline 10 & & Frontal Lobe & + & + & n.a. & 21 \\
\hline 11 & & Frontal Lobe & + & - & + & n.a. \\
\hline 12 & & Frontal Lobe & + & + & + & n.a, \\
\hline 13 & & Frontal Lobe & + & + & - & 18 \\
\hline 14 & & Frontal Lobe & + & + & + & 9 \\
\hline 15 & & Frontal Lobe & + & + & n.a. & 14 \\
\hline 16 & & Frontal Lobe & + & + & + & n.a \\
\hline 17 & & Frontal Lobe & + & + & + & 22 \\
\hline 18 & & Frontal Lobe & + & + & - & 1 \\
\hline 19 & & Frontal Lobe & + & + & + & 22 \\
\hline 20 & & Frontal Lobe & + & n.a & n.a & 54 \\
\hline 21 & & Frontal Lobe & + & + & + & 1 \\
\hline 22 & & Frontal Lobe & + & + & n.a & 1 \\
\hline 23 & & Frontal Lobe & + & + & + & 2 \\
\hline 24 & & Frontal Lobe & + & - & + & n.a. \\
\hline 25 & & Frontal Lobe & + & - & + & 5 \\
\hline 26 & & Frontal Lobe & + & + & n.a & 10 \\
\hline
\end{tabular}

Cite this article: Ravindra Pramod D, Chandrasekhar YBVK, Manas P, I Satish R, Phanithi Prakash B. Glioblastoma and Glioblastoma with 


\begin{tabular}{|c|c|c|c|c|c|}
\hline 27 & Frontal Lobe & + & + & + & 14 \\
\hline 28 & Frontal Lobe & + & + & - & n.a. \\
\hline 29 & Frontal Lobe & + & + & n.a & 23 \\
\hline 30 & Frontal Lobe & + & + & + & 31 \\
\hline 31 & Frontal Lobe & + & + & n.a & 17 \\
\hline 32 & Frontal Lobe & + & + & n.a & 7 \\
\hline 33 & Frontal Lobe & + & + & + & 20 \\
\hline 34 & Frontal Lobe & + & + & - & 15 \\
\hline 35 & Frontal Lobe & + & + & + & 14 \\
\hline 36 & Frontal Lobe & n.a & + & - & 9 \\
\hline 37 & Frontal Lobe & + & n.a. & + & 11 \\
\hline 38 & Frontal Lobe & + & + & n.a. & n.a. \\
\hline 39 & Frontal Lobe & + & + & + & 7 \\
\hline 40 & Frontal Lobe & + & - & + & 6 \\
\hline
\end{tabular}

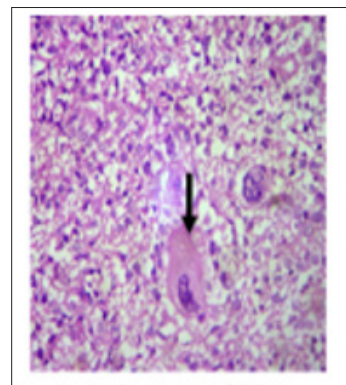

A $\mathrm{H}$ and $\mathrm{E}$ staining of $\mathrm{GBM}$

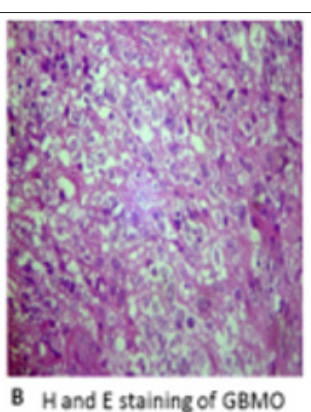

B $\mathrm{H}$ and $\mathrm{E}$ staining of GBMO
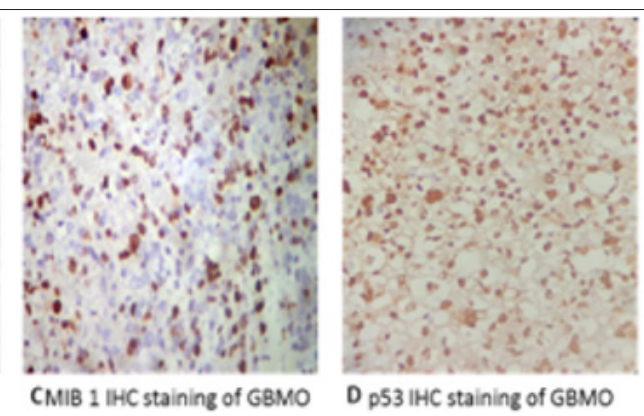

Figure 1: $\mathrm{H}$ and $\mathrm{E}$ staining of glioblastoma multiformae (GBM) (A) and glioblastoma with oligodendroglial component (GBMO) (B) Immunohistochemical staining of MIB1 (C) and p53 (D) in GBMO (40X).

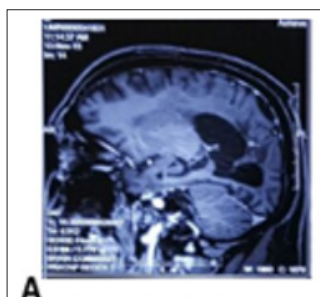

A

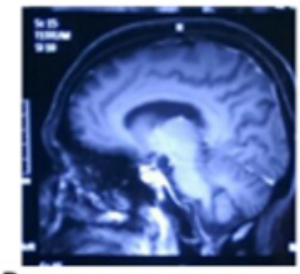

B Post operative GBM

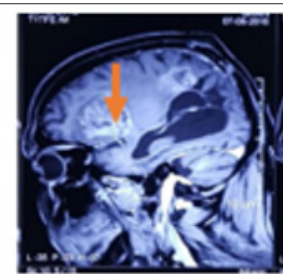

Progressive fumor ( 9 months)

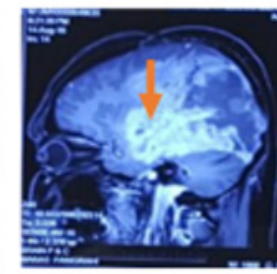

Progressive tumor ( 1 year)

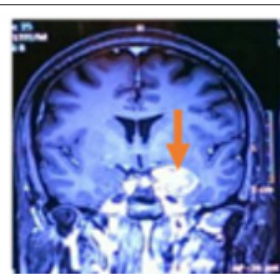

C Pre operative GBMO

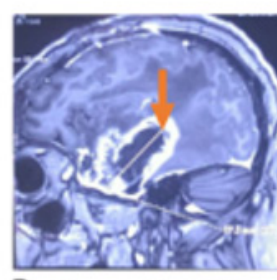

D Pre operative GBMO

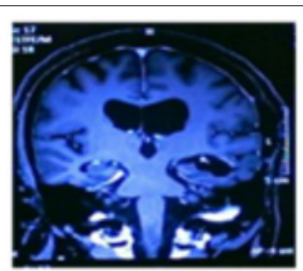

Post operative MRI ( 3 years)

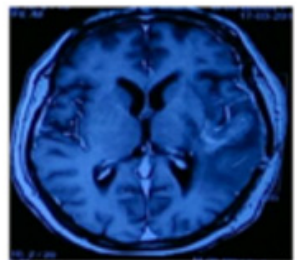

Post operative MRI (1 year)

Figure 2: Representative follow up MRI images of pre and post-operative glioblastoma and glioblastoma with oligodendroglial component.

a) Post-operative glioblastoma with observed progression after 9 months of resection.

b) Post-operative GBM with observed recurrence at occipital lobe after 1 year of resection.

c) Pre-Operative MRI scan image of occipital glioblastoma with oligodendroglial component and post-operative image taken after 3 years. D: Pre- operative MRI scan image of GBMO and post-operative MRI scan image taken after 1 year. Visible tumor has been marked by an arrow. 


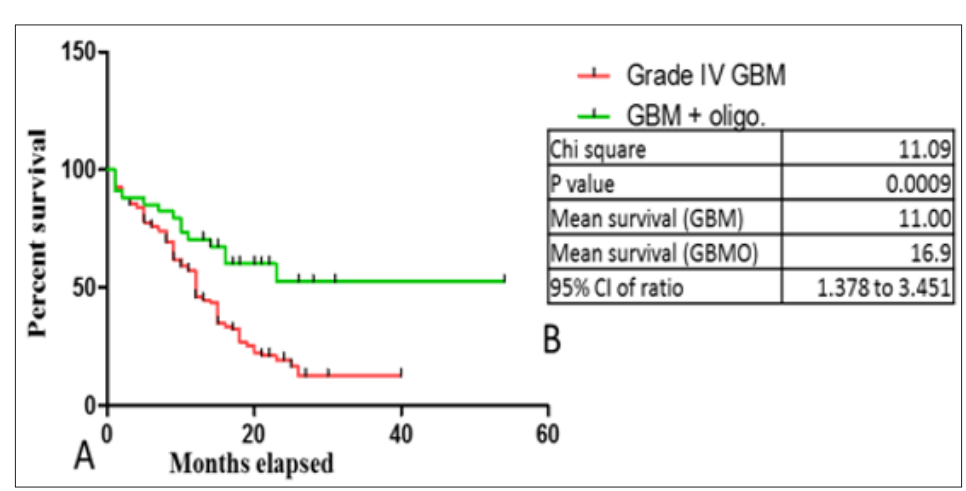

Figure 3: Kaplan-Meier survival curve for patients with glioblastoma and glioblastoma with oligodendroglial component (A). Patients' having glioblastoma + oligodendroglial component $(n=36)$ were having significantly longer survival than patients' with glioblastoma $(n=138)(B, p=0.0009)$.

\section{Discussion}

The aim of present study was to prognostically evaluate role of oligodendroglial component in survival of glioblastoma multiformae patients. There have been few studies reporting involvement of oligodendroglial component in glioblastomas and is defined by specific molecular alterations $[1,9,10]$. Glioblastomas with oligodendroglioma is genetically heterogeneous and reported to represent $4 \%$ of glioblastoma multiformae. A study has reported LOH on $1 p$ which is linked with better prognosis in anaplastic oligodendrogliomas and increased chemo sensitivity [11]. Other evidence however claims a higher incidence (17\%) than later [12]. There are controversies about survival outcomes of glioblastoma with oligodendroglial component as some studies claim better survival for patient's with glioblastoma with oligo component $[4,10,12,13]$ while some acclaim no favorable prognostic values [68]. In our study, out of total 178 cases, oligodendroglial component was confirmed in 40 cases (Table 1). This validates no frequent occurrence of glioblastomas with oligodendroglial component. We found glioblastoma patients with oligodendroglial component had better survival than previous one and is statistically significant Hazard ratio: $2.01(\mathrm{p}=0.0009$, Chi square $=11.09,95 \% \mathrm{CI}$ of ratio $=1.37$ to 3.4 ) (Figure 2).

It is apparent that the probability of deaths in GBM group was 2.01 times more than in GBMO group. Glioblastoma patients were found with 11 mean survival months while GBM with oligo component patients were found to have 16.9 months of mean survival. We have studied total of 40 GBMO cases (Table 1). Survival details were not available with 04 cases. We also found anatomic origin of tumor. In 40 cases, 13 found to be at frontal lobe, 06 at parietal lobe, 16 at temporal lobe and 04 at occipital lobe. Here, we observed non uniform distribution of tumor in brain mainly dominated by frontal and temporal lobe. Out of total subjects studied, 20 have been reported to survive for more than 2 years. In our group, we observed $50 \%$ of patients were older than 50 years at the time of diagnosis while previous studies $[1,14]$ claims this percentage to be more than $80 \%$. We have also studied for MIB1 and p53 expression profile in our group. Out of 40 GBMO cases, 22 were found to be positive with p53 overexpression while 4 cases were negative.

Details were not available with 10 cases. Overall, we found p53 positivity in $55 \%$ of cases. Recent reports claims p53 positivity in more than $70 \%$ of cases and is associated with IDH1 mutations $[10,15]$. Of total $40 \mathrm{GBMO}$ cases, $38(95 \%)$ were found to be positive for MIB1 staining while other reports claims this positivity to be as low as 31\% [16] and do not possess prognostic significance [17]. We found necrosis to be prevalent in 34 (85\%) cases. 2 cases were found to be negative while data was not available for 4 cases. Previous reports support for presence of necrosis in GBMO [13] and has been correlated with less favorable prognosis in anaplastic oligoastrocytoma $[18,19]$. GBMOs are being reported with varying frequencies of IDH mutations and lower expression of MGMT. Clinically, the IDH mutations were associated with increased overall survival. The differences have been noted in pattern of this signature marker in conventional GBM and GBMO. Nearly 20\% of GBMO were reported with IDH mutations while this percentage was sored up to nearly $90 \%$ in secondary GBM. More understating of it can provide the molecular, heterogeneous and clinical profile of GBMO [10,14]. Oligoastrocytic tumors with necrosis have been designated as glioblastomas with oligodendroglial component by WHO 2007 classification [1]. On pathological scale, GBMOs are defined as anaplastic astrocytic tumors with necrosis [20,21] and develops from malignant transformation of low-grade glial tumors. Collectively, GBMO entity was associated with better survival as compared to GBM with overexpression of p53 and MIB-1. So, a combined approach to look for associated genetic mutations is necessary to underline its intrinsic nature as prognostic marker.

\section{Funding}

Authors thank financial assistance from Department of Science and technology (DST- India) (Grant no: SB/EMEQ-257/2013, SR/CSRI/196/2016), Department of Biotechnology (DBT-India) (Grant No. BT/PR18168/MED/29/1064/2016, BT/PR13111/ MED/29/149/2009) and University with potential for excellence (UPE-India) (Grant no: UH/UGC/UPE-2/Interface studies/ 
Research Projects/B1.4, UH/UPE-2/28/2015) for lab funding. RDP thankful to Department of Biotechnology (DBT-India) (Award no: DBT JRF/2011-12/95) for student fellowship.

\section{Acknowledgements}

The authors acknowledge KFRC, KIMS for the ethical permission.

\section{Ethical approval}

The present studies involving human participants were approved by institutional ethics committee (ICE), University of Hyderabad and KIMS Foundation Research Centre (KFRC), KIMS, Secunderabad, India. All subjects participating were completely anonymized.

\section{Informed consent}

Informed consent was obtained from all individual participants included in the study.

\section{References}

1. Louis DN, Ohgaki H, Wiestler OD, Cavenee WK, Burger PC, (2007) et al. The 2007 WHO classification of tumours of the central nervous system. Acta neuropathologica 114(2): 97-109.

2. Homma T, Fukushima T, Vaccarella S, Yonekawa Y, Di Patre PL, et al. (2006) Correlation among pathology, genotype, and patient outcomes in glioblastoma. J Neuropathol Exp Neurol 65(9): 846-854.

3. Salvati M, Formichella AI, D Elia A, Brogna C, Frati A, et al. (2009) Cerebral glioblastoma with oligodendrogliomal component: analysis of 36 cases. Journal of neuro-oncology 94(1): 129-134.

4. Vordermark D, Ruprecht K, Rieckmann P, Roggendorf W, Vince GH, et al. (2006) Glioblastoma multiforme with oligodendroglial component (GBMO): favorable outcome after post-operative radiotherapy and chemotherapy with nimustine (ACNU) and teniposide (VM26). BMC cancer 6: 247.

5. Appin CL, Gao J, Chisolm C, Torian M, Alexis D, et al. (2013) Glioblastoma with oligodendroglioma component (GBM-0): molecular genetic and clinical characteristics. Brain Pathol 23(4): 454-461.

6. Pinto LW, Araujo MB, Vettore AL, Wernersbach L, Leite AC, et al. (2008) Glioblastomas: correlation between oligodendroglial components, genetic abnormalities, and prognosis. Virchows Arch 452(5): 481-490.

7. Nakamura H, Makino K, Kuratsu J (2011) Molecular and clinical analysis of glioblastoma with an oligodendroglial component (GBMO). Brain tumor pathology 28(3): 185-190.

8. Hegi ME, Janzer RC, Lambiv WL, Gorlia T, Kouwenhoven MC, et al. (2012) Presence of an oligodendroglioma-like component in newly diagnosed glioblastoma identifies a pathogenetically heterogeneous subgroup and lacks prognostic value: central pathology review of the EORTC_26981/ NCIC_CE.3 trial. Acta neuropathologica 123(6): 841-852.

\section{ISSN: 2574-1241}

DOI: 10.26717/BJSTR.2018.09.001828

P Prakash Babu. Biomed J Sci \& Tech Res

This work is licensed under Creative Commons Attribution 4.0 License

Submission Link: https://biomedres.us/submit-manuscript.php
9. Hinrichs BH, Newman S, Appin CL, Dunn W, Cooper L, et al. (2016) Farewell to GBM-O: Genomic and transcriptomic profiling of glioblastoma with oligodendroglioma component reveals distinct molecular subgroups. Acta neuropathologica communication 4:4.

10. Wang Y, Li S, Chen L, You G, Bao Z, et al. (2012) Glioblastoma with an oligodendroglioma component: distinct clinical behavior, genetic alterations, and outcome. Neuro-oncology 14(4): 518-525.

11. Kraus JA, Lamszus K, Glesmann N, Beck M, Wolter M, et al. (2001) Molecular genetic alterations in glioblastomas with oligodendroglial component. Acta neuropathologica. 101(4): 311-320.

12. He J, Mokhtari K, Sanson M, Marie Y, Kujas M, et al. (2001) Glioblastomas with an oligodendroglial component: a pathological and molecular study. J Neuropathol Exp Neurol 60(9): 863-871.

13. Kanno H, Nishihara H, Narita T, Yamaguchi S, Kobayashi H, et al. (2012) Prognostic implication of histological oligodendroglial tumor component: clinicopathological analysis of 111 cases of malignant gliomas. Plos one 7e41669.

14. Okamoto Y, Di Patre PL, Burkhard C, Horstmann S, Jourde B, et al. (2004) Population-based study on incidence, survival rates, and genetic alterations of low-grade diffuse astrocytomas and oligodendrogliomas. Acta neuropathologica 108(1): 49-56.

15. Myung JK, Cho HJ, Kim H, Park CK, Lee SH, et al. (2014) Prognosis of Glioblastoma with Oligodendroglioma Component is Associated With the IDH1 Mutation and MGMT Methylation Status. Transl Oncol 7(6): 712-719.

16. Hata N, Suzuki SO, Murata H, Hatae R, Akagi Y, et al. (2015) Genetic analysis of a case of glioblastoma with oligodendroglial component arising during the progression of diffuse astrocytoma. Pathol Oncol Res 21(3): 839-843.

17. Jiang H, Ren X, Wang J, Zhang Z, Jia W (2014) Short-term survivors in glioblastomas with oligodendroglioma component: a clinical study of 186 Chinese patients from a single institution. Journal of neuro-oncology 116(2): 395-404.

18. Smith SF, Simpson JM, Brewer JA, Sekhon LH, Biggs MT, et al. (2006) The presence of necrosis and/or microvascular proliferation does not influence survival of patients with anaplastic oligodendroglial tumours: review of 98 patients. Journal of neuro-oncology 80(1): 75-82.

19. Miller CR, Dunham CP, Scheithauer BW, Perry A (2006) Significance of necrosis in grading of oligodendroglial neoplasms: a clinicopathologic and genetic study of newly diagnosed high-grade gliomas. Journal of clinical oncology 24(34): 5419-5426.

20. Klink B, Schlingelhof B, Klink M, Stout Weider K, Patt S, et al. (2010) Glioblastomas with oligodendroglial component - common origin of the different histological parts and genetic subclassification. Anal Cell Pathol (Amst) 33(1): 37-54.

21. Hartmann C, von Deimling A (2009) Molecular pathology of oligodendroglial tumors. Recent Results Cancer Res 171: 25-49.

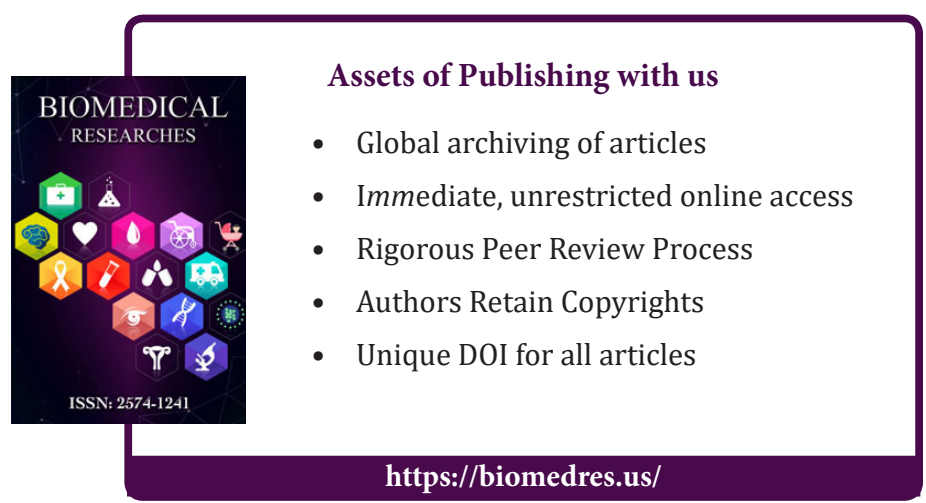

Cite this article: Ravindra Pramod D, Chandrasekhar YBVK, Manas P, I Satish R, Phanithi Prakash B. Glioblastoma and Glioblastoma with Oligodendroglial Component: a Histological and Prognostic Overview Running Head: pathology and GBM and GBMO Biomed J Sci\&Tech Res

9(4)-2018. BJSTR. MS.ID.001828. DOI: 10.26717/ BJSTR.2018.09.001828. 